This is an author version of an abstract (from the conference Fourth annual conference of the European Association of Psychosomatic Medicine (EAPM): Transforming Health with Evidence and Empathy.

Citation for the published publication:

M. Nordin, R. Bucher, Nina Lind, S. Nordin. (2016) Is there a bidirectional relationship between sleep and asthma and allergy in a general population?. Presenterades vid: Fourth annual conference of the European Association of Psychosomatic Medicine (EAPM): Transforming Health with Evidence and Empathy, Luleå, 160616-160618, The European Association for Psychosomatic Medicine (EAPM). http://eapm2016.com/

This abstract was also published in the Journal of Psychosomatic research: http://dx.doi.org/10.1016/j.jpsychores.2016.03.186

Published with permission from: Elsevier.

Standard set statement from the publisher:

(C) Elsevier, 2016 This manuscript version is made available under the CC-BY-NC-ND 4.0 license http://creativecommons.org/licenses/by-nc-nd/4.0/

Epsilon Open Archive http://epsilon.slu.se 


\title{
Is there a bidirectional relationship between sleep and asthma and allergy in a general population?
}

\author{
M. Nordin ${ }^{a}$, R. Bucher ${ }^{a}$, Nina Lind ${ }^{b}$, S. Nordin ${ }^{a}$ \\ ${ }^{a}$ Department of Psychology, Umeå University, Umeå, Sweden \\ b Department of Economics, Swedish University of Agricultural Science,Uppsala, Sweden
}

Background: The presented study aims at examining the relationship between sleep, on the one hand, and allergic asthma, non-allergic asthma, allergic rhinitis, and atopic dermatitis, on the other hand. These afflictions are highly comorbid, and a common denominator is nasal congestion and itching peaking at night, which may disturb sleep. However, previous research has suggested an alternative relationship in which insomnia precedes asthma and allergy. The objective is thus to explore whether there is a bidirectional relationship between sleep and asthma/allergy.

Method: The prospective population based Västerbotten Environmental Health Study (VEHS) was used. Among 8520 invited individuals aged 18-79 years, $3406(40 \%)$ responded in 2010 (T1) to a questionnaire focusing on environmental hypersensitivity, asthma, and allergy. Among 3181 of these participants who still were still alive and living in Västerbotten, 2336 (73\%) completed the questionnaire again in 2013 (T2). Self-reported physician-based diagnoses on allergic and nonallergic asthma, allergic rhinitis, and atopic dermatitis were used for identifying the four afflictions. Sleep was operationalized as insomnia by using the sleep quality and non-restorative sleep scales of the Karolinska Sleep Questionnaire (KSQ). Logistic regression analysis was used to calculate odds ratio (OR) and $95 \%$ confidence intervals (CI). Age, sex, stress, burnout, depression, and anxiety, assessed with validated questionnaire instruments, were tested for confounding. Referents were free of any asthma or allergy.

Results: At T2, 30 participants had developed allergic asthma, 42 non-allergic asthma, 64 allergic rhinitis, and 19 atopic rhinitis since T1. No bidirectional relationships were found between sleep and asthma, allergic rhinitis, or atopic dermatitis. However, allergic asthma was found to precede insomnia (OR 1.64, 95 \% CI 1.06-2.55). The association turned insignificant though when adjusting for either stress or burnout, possibly indicating a mediating association. Age, sex, depression and anxiety did not alter the crude relationship notably.

Conclusion: No bidirectional relationships were found between sleep and asthma/allergy. People with allergic asthma were at higher risk for insomnia though. However, this elevated risk may be due to stress. Given the fact that stress and poor sleep can lead to ill-health and lower life quality, these factors are important for health care personnel and patients with allergic asthma to observe. 